\title{
Activation Mode Effects on the Shear Bond Strength of Dual-cured Resin Cements
}

\author{
AL Faria-e-Silva • MM Fabião \\ VG Arias • LRM Martins
}

\section{Clinical Relevance}

The light activation of dual-cured resin cements is essential for improved shear bond strength. Bond strength is low in the first minutes following insertion of a resin cement and increases over time. Under clinical conditions, care should be taken to limit occlusal stress immediately after luting an indirect restoration.

\section{SUMMARY}

Objectives: This study evaluated the immediate (10-minute) and delayed (24-hour) bond strength of dual-cured resin cements that are light-activated either immediately or delayed (after five minutes) or chemically-activated only. Materials and Methods: Three dual-cured resin cements were evaluated: RelyX ARC, Panavia F and Enforce. Cylinders of resin cement were built up

*André Luis Faria-e-Silva, DDS, MS, PhD, Federal University of Sergipe, Department of Dentistry, Aracaju, SE, Brazil

Mayra Melo Fabião, DDS, graduate student, State University of Campinas, Piracicaba, SP, Brazil

Vanessa Gallego Arias, DDS, MD, PhD, São Leopoldo Mandic School of Dentistry, Department of Restorative Dentistry, Campinas, SP, Brazil

Luis Roberto Marcondes Martins, DDS, MD, PhD, State University of Campinas, Department of Restorative Dentistry, Piracicaba, SP, Brazil

*Reprint request: Rua Cláudio Batista S/N-Bairro Sanatório, Aracaju, SE 49060-100, Brazil; e-mail: andrelfsilva@hotmail.com DOI: $10.2341 / 10-051-\mathrm{L}$ over resin composite blocks following the manufacturers' instructions for each luting agent. The cements were mixed, inserted into the molds and light-activated either immediately or after five minutes (delayed light activation). When no light activation was performed, the materials were protected from light exposure until testing. Half of the samples were tested at 10 minutes; the remaining samples were stored at $100 \%$ relative humidity for 24 hours when they were then tested $(n=10)$. Data were submitted to three-way ANOVA and post-hoc Tukey's tests $(\alpha=0.05)$. The failure mode was evaluated under SEM. Results: RelyX ARC presented the highest values of shear bond strength, followed by Enforce. Panavia F showed the lowest values. Both immediate and delayed light activation caused the cements to present the highest means of shear bond strength. There was an improvement in bond strength after 24 hours of storage. Conclusions: RelyX ARC produced the highest bond strength, which was improved by light activation and storage for 24 hours. 


\section{INTRODUCTION}

Traditionally, tooth preparations receiving indirect restoration involve the removal of considerable tooth structure and, as more structure is removed, a tooth will be less resistant to fracture. ${ }^{1}$ Advances in bonding procedures and improvements in adhesive cementation enable more conservative preparation designs. ${ }^{2}$ However, the clinical performance of these adhesively cemented indirect restorations depends on bonding of the luting agent to both the tooth and the restorative material. ${ }^{3-4}$

For this purpose, resin-based luting agents are commonly used in dentistry. However, proper polymerization of the luting material is essential for the clinical success of restorations. Thus, low values for the degree of conversion (DC) for these materials might reduce retention of the indirect material, or the post, and contribute to restoration failures..$^{5-6}$

Dual-cured resin cements were introduced to combine the favorable characteristics of self- and light-cured agents. The rationale was to develop a material with extended working time and which is capable of reaching high DC in either the presence or absence of light. During the cementation of posts and thick indirect restorations, exposed marginal areas can largely benefit from photoactivation, as they are readily accessible to the curing light; however, a significant reduction in the intensity of the curing light might occur due to reflecting and scattering effects. ${ }^{7.8}$ Therefore, in some situations, the polymerization reaction may be mainly activated by a chemical mechanism (self-cure). However, it has been shown that the polymerization of dual-polymerized resin cements might depend on their exposure to light for achieving better mechanical properties. ${ }^{9-10}$

Despite the importance of proper polymerization of resin cements with regards to the retention of indirect restorations, bond strength evaluations are conventionally performed at 24 hours or longer after restorative procedures. ${ }^{11-13}$ Clinically, however, the interfaces are subjected to stress immediately upon completion of cementation. In this approximate 10-minute period, it is unclear whether resin cement reaches proper polymerization and satisfactory bonding to both restorative material and tooth surface, mainly in the absence of light activation. In addition, some manufacturers recommend that clinicians remove the excess resin cement approximately five minutes after seating the restoration. This period is required to increase the viscosity of resin cements, making excess removal easier. Then, the resin cement is light-cured after this delay period. However, the effect of this delay period has not been determined and warrants further investigation.

Thus, the current study evaluated the immediate (10minute) and delayed (24-hour) bond strength of dualcured resin cements to resin composite blocks activated by one curing mode: immediate or delayed light activation, or chemical activation only. The null hypothesis was that the bond strength would not be dependent on either the curing mode or the time of evaluation.

\section{METHODS AND MATERIALS}

One-hundred and eighty light-activated resin composite discs (2-mm thick and $6 \mathrm{~mm}$ in diameter-A2 shade-Z250, 3M/ESPE, St Paul, MN, USA) were prepared to simulate overlying laboratory-processed resin composite restorations. ${ }^{14}$ The resin composite was inserted in $1 \mathrm{~mm}$-thick increments, and each increment was polymerized for three minutes in a laboratory light-curing unit, Edglux (EDG Ltda, São Carlos, SP, Brazil). After light-activation of each increment, the resin composite cylinder was submitted to additional light-activation for seven minutes. The surface of each pre-cured resin disc scheduled for bonding was wetground with 600 -grit silicon carbide abrasive paper for 15 seconds. The surface was then sandblasted with 50 $\mu \mathrm{m}$ aluminum oxide particles for 10 seconds, air pressure: 80 psi at a distance of $1.5 \mathrm{~cm}$ from the tip. ${ }^{15}$

The resin-based luting systems used in the current study are shown in Table 1 . In order to obtain a resin cement cylinder for shear testing, cylindrical polyvinyl

\begin{tabular}{|c|c|c|}
\hline Material & Manufacturer & Composition* \\
\hline Enforce & $\begin{array}{l}\text { Dentsply } \\
\text { Petrópolis, RJ, Brazil }\end{array}$ & $\begin{array}{l}\text { Base: Bis-GMA, TEGDMA, CQ, EDAB, BHT and DHEPT } \\
\text { Catalyst: Bis-GMA, BHT, EDAB, TEGDMA and BPO }\end{array}$ \\
\hline RelyX ARC & $\begin{array}{l}\text { 3M ESPE } \\
\text { St Paul, MN, USA }\end{array}$ & $\begin{array}{l}\text { Paste A: Bis-GMA, TEGDMA, dimethacrylate polymer, CQ, amine } \\
\text { Paste B: Bis-GMA, TEGDMA, dimethacrylate polymer, BPO }\end{array}$ \\
\hline Panavia F & $\begin{array}{l}\text { Kuraray Co } \\
\text { Osaka, Japan }\end{array}$ & $\begin{array}{l}\text { Paste A: 10-MDP, hydrophobic and hydrophilic dimethacrylate, CQ, BPO } \\
\text { Paste B: Hydrophobic and hydrophilic dimethacrylate, DHEPT, T-isopropylic benzenic } \\
\text { sodium sulfinate }\end{array}$ \\
\hline ED Primer & $\begin{array}{l}\text { Kuraray Co } \\
\text { Osaka, Japan }\end{array}$ & $\begin{array}{l}\text { Primer A: HEMA, 10-MDP, NM-aminosalicilic acid, DHEPT, water } \\
\text { Primer B: NM-aminosalicilic acid, T-isopropylic benzenic sodium sulfinate, DHEPT, } \\
\text { water }\end{array}$ \\
\hline
\end{tabular}


siloxane impression material molds (Aquasil Extra Low Viscosity, Dentsply DeTrey, Konstanz, Germany) with internal diameters of $2 \mathrm{~mm}$ and about $2 \mathrm{~mm}$ in height were placed over the previously prepared surfaces of the resin composite. ${ }^{16-17}$ All of the luting systems were manipulated and applied to the composite surfaces according to the manufacturers' recommendations.

RelyX ARC-The RelyX Ceramic Primer was applied on the sandblasted surface of the resin composite and air-dried for five seconds. Equal amounts of the A and B pastes were mixed for 15 seconds and placed into the mold.

Panavia F-One drop each of Clearfil Porcelain Bond Activator (Kuraray Dental Co, Okayama, Japan) and Clearfil SE Bond Primer (Kuraray Dental Co) were mixed. The mixture was applied to the sandblasted surface, and a gentle air stream was applied for 10 seconds. $\mathrm{A}$ and $\mathrm{B}$ pastes of Panavia $\mathrm{F}$ were mixed for 20 seconds and placed into the mold.

Enforce-Equal amounts of Primer and Activator of the silane agent (Dentsply Ind e Comércio ltda, Petrópolis, RJ, Brazil) were mixed five minutes prior to use. Then, the silane was applied on the resin composite surface and air-dried for 10 seconds. Next, the adhesive system (Prime\&Bond NT) was applied, air-dried for five seconds, and light-cured for 10 seconds. For the delayed light-activated and self-cured groups, one drop of self-cure activator was mixed with one drop of adhesive before application. Finally, the catalyst and base pastes were mixed for 20 seconds and inserted into the mold.

The following polymerization scenarios were tested for all resin cements.

Immediate Light Activation: The resin cements were light-activated immediately after mixing and placed into the mold for the time periods recommended by the manufacturers: 20 seconds (Panavia F), 30 seconds (Enforce) and 40 seconds (RelyX ARC).

Delayed Light Activation: The luting agents were light-activated five minutes after beginning the mixing procedure for the same times as described above.

Chemical Activation: No light activation was performed and polymerization relied on self-activation only.
Light activation was performed through the nonbonded surface of each resin composite disc, simulating the clinical situation in which the resin cement is indirectly light-activated. All light polymerization procedures were performed with an Optilux 501 (Demetron Kerr, Orange, CA, USA), using an output intensity of approximately $650 \mathrm{~mW} / \mathrm{cm}^{2}$. After 10 minutes, the molds were removed to expose the resin cement cylinder. Half of the samples were immediately tested, and the remainder were stored in $100 \%$ relative humidity for 24 hours before they were tested.

Each resin composite block was attached to a testing device, and the resin cement cylinders were tested in the testing machine (EMIC DL 2000, São José dos Pinhais, PR, Brazil). A shear load was applied to the base of the resin cement cylinder with a thin wire $(0.2$ $\mathrm{mm}$ in diameter), at a crosshead speed of 0.5 $\mathrm{mm} /$ minute, until failure. ${ }^{16-17}$ Shear bond strengths were calculated and expressed in MPa. Data were submitted to three-way ANOVA and post-hoc Tukey tests at a 95\% confidence level. The factors evaluated were resin cement, mode of activation and testing time.

After testing, debonded samples were mounted on aluminum stubs, gold-sputter coated (SCD 050, Baltec, Vaduz, Liechtenstein) and examined under scanning electron microscopy (JSM-5600LV, JEOL, Tokyo, Japan). The failure mode was classified into one of the following types: Type I-cohesive failure of substrate; Type II-cohesive failure of resin cement; Type IIIadhesive failure between the luting agent and substrate; and Type IV—mixed failure.

\section{RESULTS}

ANOVA showed significant effects for the factors "resin cement" $(p<0.0001)$, "mode of activation" $(p<0.0001)$ and "testing time" $(p<0.0001)$. However, there was no significant effect for any interactions among the factors. Comparisons according to the Tukey's test are presented in Tables 2 through 4. RelyX ARC presented the highest shear bond strength and Panavia F the lowest. There was no difference between immediate and delayed-light activation. The lowest values of bond strength were obtained when the resin cements were not light-activated. Specimens tested at 24 hours exhibited significantly higher shear bond strengths than those tested at 10 minutes.

\begin{tabular}{|ccccc|}
\hline \multicolumn{5}{|c|}{ Table 2: Means in MPa $( \pm S D)$ of Shear Bond Strength for Resin Cement and Mode of Activation Factors } \\
\hline \multicolumn{5}{|c|}{ Resin Cement } \\
\hline Mode of Activation & RelyX ARC & Enforce & Panavia F & Pooled Average \\
\hline Immediate light-activation & $19.5(6.0)$ & $14.7(5.4)$ & $12.0(3.9)$ & $15.4(5.9)$ A \\
Delayed light-activation & $17.0(5.1)$ & $14.5(4.3)$ & $12.1(3.5)$ & $14.5(4.7)$ A \\
Without light-activation & $12.9(4.1)$ & $8.7(3.5)$ & $8.1(3.7)$ & $9.9(4.3)$ B \\
Pooled average & $16.5(5.7)$ A & $12.6(5.2)$ B & $10.7(4.1)$ C & \\
\hline For the pooled averages, means followed by different letters differ among them by the Tukey's test at the 95\% confidence level. \\
\hline
\end{tabular}




\begin{tabular}{|ccccc|}
\hline \multicolumn{5}{|c|}{ Table 3: Means in MPa $( \pm S D)$ of Shear Bond Strength for Resin Cement and Testing Time Factors } \\
\hline \multicolumn{5}{|c|}{ Resin Cement } \\
\hline Testing Time & RelyX ARC & Enforce & Panavia F & Pooled Average \\
\hline 10 minutes & $13.5(3.9)$ & $9.8(3.4)$ & $7.8(2.6)$ & $10.3(4.1)$ B \\
24 hours & $19.5(5.8)$ & $15.5(5.1)$ & $13.7(3.0)$ & $16.2(5.3)$ A \\
Pooled average & $16.5(5.7)$ A & $12.6(5.2)$ B & $10.7(4.1)$ C & \\
\hline For the pooled averages, means followed by different letters differ among them by the Tukey's test at the 95\% confidence level. \\
\hline
\end{tabular}

\begin{tabular}{|ccccc|}
\hline \multicolumn{5}{|c|}{ Table 4: Means in MPa $( \pm S D)$ of Shear Bond Strength for Mode of Activation and Testing Time Factors } \\
\hline \multicolumn{5}{|c|}{ Mode of Activation } \\
\hline Testing Time & $\begin{array}{c}\text { Immediate } \\
\text { Light-activation }\end{array}$ & $\begin{array}{c}\text { Delayed } \\
\text { Light-activation }\end{array}$ & $\begin{array}{c}\text { Without } \\
\text { Light-activation }\end{array}$ & Pooled Average \\
\hline 10 minutes & $11.6(3.7)$ & $12.1(3.6)$ & $7.3(3.1)$ & $10.3(4.1) \mathrm{B}$ \\
24 hours & $19.2(5.4)$ & $17.0(4.6)$ & $12.5(3.7)$ & $16.2(5.3) \mathrm{A}$ \\
Pooled average & $15.4(5.9) \mathrm{A}$ & $14.5(4.7) \mathrm{A}$ & $9.9(4.3) \mathrm{B}$ & \\
\hline For the pooled averages, means followed by different letters differ among them by the Tukey's test at the 95\% confidence level. \\
\hline
\end{tabular}

Figures 1 and 2 show the failure modes of tested specimens at 10 minutes and 24 hours, respectively. All luting systems tested at 24 hours and RelyX ARC tested at 10 minutes, mainly for light-cured groups, presented predominantly Type I failures (cohesive of substrate). Few Type II failures were observed, and these occurred mainly in the cements that were only self-activated and tested at 10 minutes. Type III failures (adhesive) occurred mainly in the Panavia F and Enforce specimens tested at 10 minutes. The Type IV failure was presented only by cements tested at 10 minutes and non-light-cured Panavia F and Enforce at 24 hours.

\section{DISCUSSION}

In the current study, resin composite blocks were used as a substrate to bond the resin cement cylinders, in contrast to the dentin substrate used in most studies. Resin-to-resin specimens were used to avoid bonding to the tooth structure, which could interfere with the bond strength values, due to dentin regional variability. ${ }^{18}$ The present results showed that both the mode of activation and testing time influenced the shear bond strength of the evaluated resin cement. Thus, the null hypothesis of the current study was rejected. Independent of testing time and mode of activation, RelyX ARC presented the highest shear bond strength means, while Panavia F presented the lowest. Disregarding a possible difference in the effectiveness of silane agents, these results may be related to mechanical interlocking provided by luting agents. Sandblasting the resin composite with aluminum oxide removes mainly the resin matrix, creating an irregular surface. ${ }^{19}$ Thus, the fundamental principle of bonding is based on the micromechanical interlocking of the resin cement with resin composite blocks. Considering that the resin cement/adhesive replaces the matrix of resin composite, the higher cohesive strength of resin cement/adhesive improves the shear bond strength. The layer created by the penetration of cement/adhesive into the sandblasted resin composite could have greater strength than the underlying, noninfiltrated sandblasted layer. This could be confirmed by the prevalence of cohesive failures of substrate for the samples that presented high shear bond strength values.

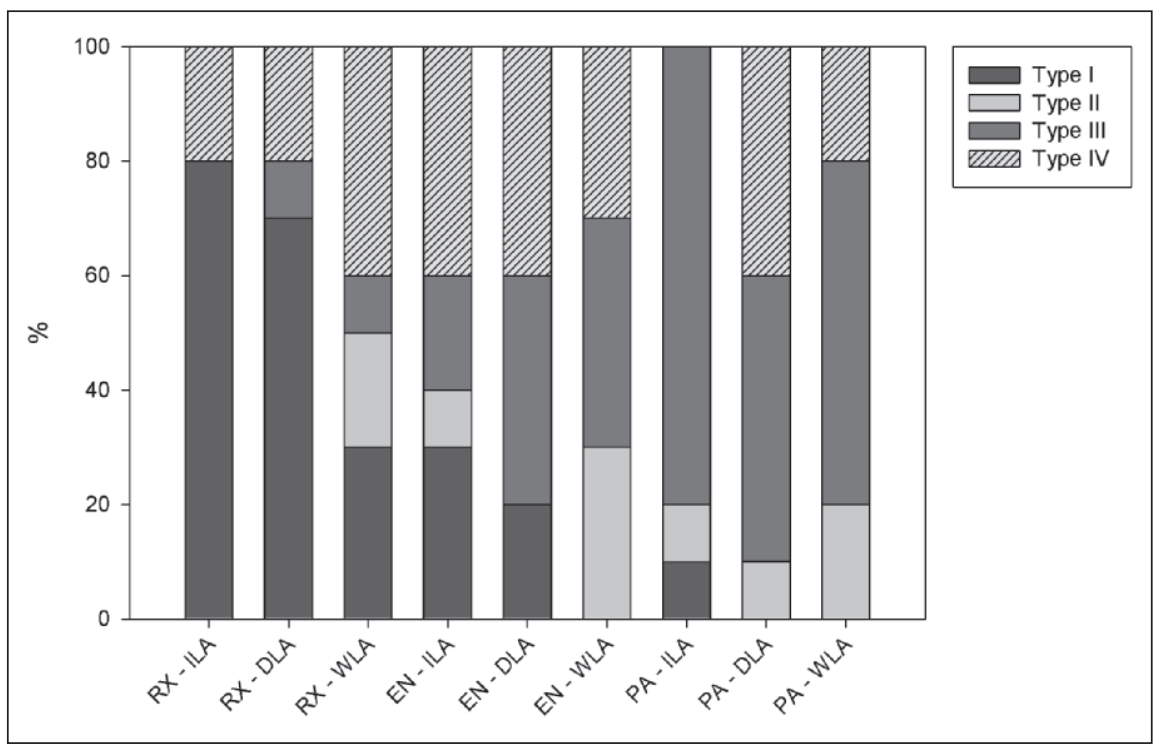

Figure 1. Proportional prevalence (\%) of failure patterns for samples tested at 10 minutes. $R X-R e l y X A R C, E N-E n f o r c e, P A-P a n a v i a$ F, ILA-Immediate light-activation, DLA-Delayed lightactivation and WLA-Without light-activation. 


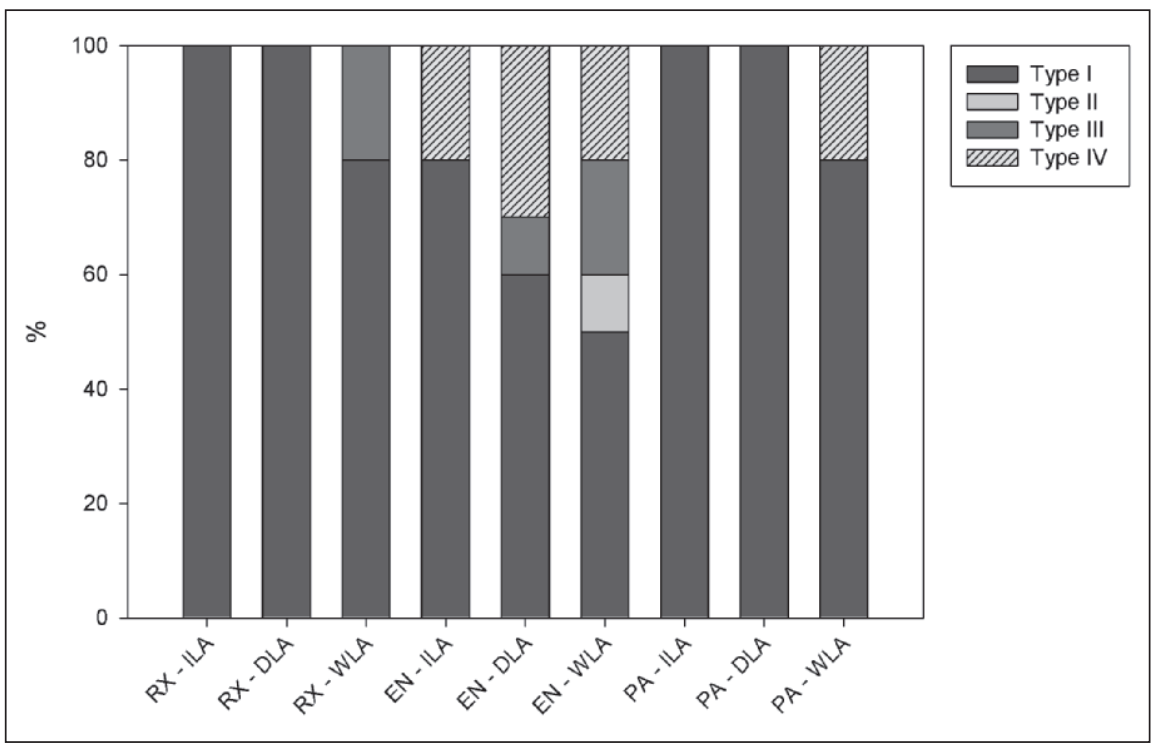

Figure 2. Proportional prevalence (\%) of failure patterns for samples tested at 24 hours. $R X-R e l y X A R C, E N-E n f o r c e, P A-P a n a v i a$ F, ILA-Immediate light-activation, DLA-Delayed lightactivation and WLA-Without light-activation.

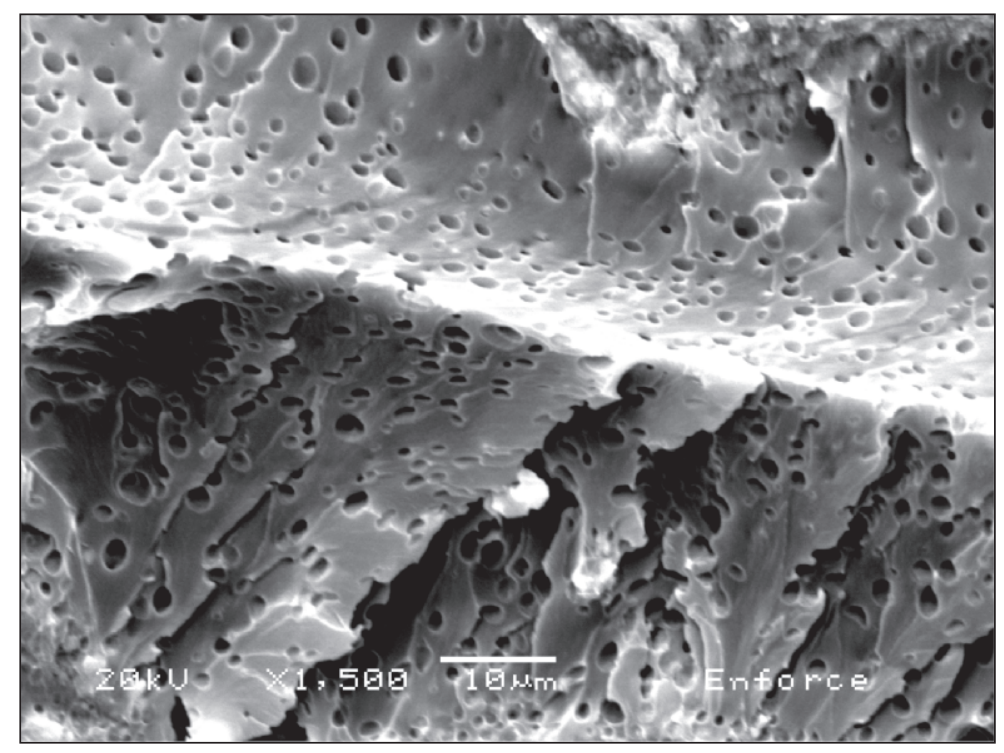

Figure 3. Fracture surface of the adhesive layer between the resin cement and substrate at no-light-cured Enforce. Note the presence of blisters in the adhesive layer formed by Prime\&Bond NT and the self-cure activator.

According to this approach, the penetration ability of the cement is fundamental to high bond strengths. Thus, RelyX ARC probably effectively penetrated into the micro-retentions created by sandblasting, due to its lower viscosity. ${ }^{20}$ In contrast to the RelyX ARC, the higher viscosity of Panavia F probably did not permit penetration into the micro-retentions, resulting in poor bond strength. Despite the high viscosity of Enforce, the manufacturer recommends that it is used with the adhesive Prime\&Bond NT. The low viscosity of this adhesive permits its penetration. However, this adhesive system presents solvents (acetone) in a composition that can reduce its cohesive strength. ${ }^{21}$ In addition, the use of a catalyst self-cure activator improves the solvent's ratio, reducing the degree of conversion of the adhesive, ${ }^{22}$ and further compromising its mechanical properties (Figure 3). This can explain the intermediate values of shear bond strength presented by Enforce.

The use of this catalyst is required to overcome the adverse chemical interaction between the simplified adhesive systems and dual-cured cements used in the absence of light activation. ${ }^{23}$ For the delayed-light activation and selfcuring activation modes, the acidic monomers of Prime\&Bond NT can react with the tertiary amine of the resin cement and prevent polymerization by self-curing components. ${ }^{24-25}$ This could compromise the bond strength of the adhesive to resin cement. Thus, the catalyst used was sufficient to prevent this incompatibility, since there was no difference between the immediate and delayed light activation of Enforce.

For all periods of evaluation, either immediate or delayed light activation created the highest values of bond strength to the evaluated resin cements. Theoretically, dual-cured resins should polymerize properly in either the presence or absence of light. In contrast to luting agents that only rely on the self-activation mechanism, there is a limit to the amount of self-polymerization promoters that can be added to dual-cured agents without impairing their working time; depending on the formulation, to some extent, the self-curing mechanism is restricted. Previous studies corroborate that dualcured luting agents are dependent on adequate light exposure to achieve higher conversion ${ }^{8,26}$ and generate better properties. ${ }^{9-10}$ Furthermore, it has been suggested that delayed light activation could improve the degree of conversion, mainly when the energy dose applied over the material is low. ${ }^{27}$ The indirect light activation used in the current study reduces the energy dose,$^{26}$ but no effects of delayed activation were observed in bond strength. It is important to emphasize that, in addition to the degree of conversion, the polymer structure is essential to the proper mechanical properties of the material. ${ }^{28}$ Thus, delayed light activation probably does not significantly affect the cohesive strength of cement, resulting in similar shear bond strengths. 
This improvement in bond strength, made by increasing the degree of conversion, may also explain the superior results obtained when the testing was accomplished at 24 hours. Increases in shear bond strength over a 24-hour period may be related to further polymerization of the resin cement, ${ }^{29}$ enhancing its bonding ability. It is difficult to determine the minimum bond strength required to support the stress generated by shrinkage polymerization and occlusal load.

Based solely on the results of the current study, it appears that light activation of cement is essential in improving the bond strength during the first minutes after cementation. However, light activation enhances the stress generated by polymerization. ${ }^{30}$ Several studies have shown that marginal defects are frequently present under bonded indirect restorations. ${ }^{31-33}$ Thus, stress reduction strategies associated with high bond strength may help to reduce these marginal defects. Following this approach, delayed light activation could be a proper strategy for extending the gel point. However, it is necessary to evaluate possible stress reduction by delayed light activation.

\section{CONCLUSIONS}

The current study revealed the following:

- RelyX ARC produced the highest shear bond strength and Panavia F the lowest.

- Both immediate and delayed light activation produced higher bond strengths than the nonlight-activated cement. There was no difference between the two periods of light activation.

- The samples tested after 24 hours of storage presented higher bond strengths than those tested at 10 minutes.

\section{Acknowledgements}

This study was supported by FAPESP grants \#2007/01006-1 and 2007/00998-0.

(Received 18 February 2010; Accepted 22 March 2010)

\section{References}

1. Fonseca RB, Correr-Sobrinho L, Fernandes-Neto AJ, Quagliatto OS \& Soares CJ (2008) The influence of the cavity preparation design on marginal accuracy of laboratoryprocessed resin composite restorations Clinical Oral Investigations 12(1) 53-59.

2. Soares PV, Santos-Filho PC, Gomide HA, Araujo CA, Martins LR \& Soares CJ (2008) Influence of restorative technique on the biomechanical behavior of endodontically treated maxillary premolars. Part II: Strain measurement and stress distribution The Journal of Prosthetic Dentistry 99(2) 114-122.
3. Soares PV, Santos-Filho PC, Martins LR \& Soares CJ (2008) Influence of restorative technique on the biomechanical behavior of endodontically-treated maxillary premolars Part I: Fracture resistance and fracture mode The Journal of Prosthetic Dentistry 99(1) 30-37.

4. Pilo R, Lewinstein I, Ratzon T, Cardash HS \& Brosh T (2008) The influence of dentin and/or metal surface treatment on the retention of cemented crowns in teeth with an increased taper Dental Materials 24(8) 1058-1064.

5. Zhang L, Magni E, Radovic I, Wang YJ, Chen JH \& Ferrari M (2008) Effect of curing modes of dual-curing luting systems and root regions on retention of translucent fiber posts in root canals The Journal of Adhesive Dentistry 10(3) 219-226.

6. Cavalcanti SC, de Oliveira MT, Arrais CA \& Giannini M (2008) The effect of the presence and presentation mode of coinitiators on the microtensile bond strength of dual-cured adhesive systems used in indirect restorations Operative Dentistry 33(6) 682-689.

7. Arrais CA, Rueggeberg FA, Waller JL, de Goes MF \& Giannini M (2008) Effect of curing mode on the polymerization characteristics of dual-cured resin cement systems Journal of Dentistry 36(6) 418-426.

8. Faria-e-Silva AL, Arias VG, Soares LE, Martin AA \& Martins LR (2007) Influence of fiber-post translucency on the degree of conversion of a dual-cured resin cement Journal of Endodontics 33(3) 303-305.

9. Yoshida K, Tsuo Y, Meng X \& Atsuta M (2007) Mechanical properties of dual-cured resin luting agents for ceramic restoration Journal of Prosthodontics 16(5) 370-376.

10. Meng X, Yoshida K \& Atsuta M (2008) Influence of ceramic thickness on mechanical properties and polymer structure of dual-cured resin luting agents Dental Materials 24(5) 594599.

11. Harnirattisai C, Kuphasuk W, Senawongse P \& Tagami J (2009) Bond strengths of resin cements to astringent-contaminated dentin Operative Dentistry 34(4) 415-422.

12. D’Arcangelo C, De Angelis F, D’Amario M, Zazzeroni S, Ciampoli C \& Caputi S (2009) The influence of luting systems on the microtensile bond strength of dentin to indirect resinbased composite and ceramic restorations Operative Dentistry 34(3) 328-336.

13. Lee JI \& Park SH (2009) The effect of three variables on shear bond strength when luting a resin inlay to dentin Operative Dentistry 34(3) 288-292.

14. Arrais CA, Giannini M, Rueggeberg FA \& Pashley DH (2007) Effect of curing mode on microtensile bond strength to dentin of two dual-cured adhesive systems in combination with resin luting cements for indirect restorations Operative Dentistry 32(1) $37-44$.

15. Soares CJ, Soares PV, Pereira JC \& Fonseca RB (2005) Surface treatment protocols in the cementation process of ceramic and laboratory-processed composite restorations: A literature review Journal of Esthetic and Restorative Dentistry 17(4) 224-235.

16. Moraes RR, Correr-Sobrinho L, Sinhoreti MA, PuppinRontani RM, Ogliari FA \& Piva E (2008) Light-activation of resin cement through ceramic: Relationship between irradiance intensity and bond strength to dentin Journal of Biomedical Materials Research Part B: Applied Biomaterials 85(1) 160-165. 
17. Faria-e-Silva AL, Fabião MM, Sfalcin RA, de Souza Meneses M, Santos-Filho PC, Soares PV \& Martins LR (2009) Bond strength of one-step adhesives under different substrate moisture conditions European Journal of Dentistry 3(4) 290 296.

18. Fawzy AS (2010) Variations in collagen fibrils network structure and surface dehydration of acid demineralized intertubular dentin: Effect of dentin depth and air-exposure time Dental Materials 26(1) 35-43.

19. D'Arcangelo C \& Vanini L (2007) Effect of three surface treatments on the adhesive properties of indirect composite restorations Journal of Adhesive Dentistry 9(3) 319-326.

20. Lee JH, Um CM \& Lee IB (2006) Rheological properties of resin composites according to variations in monomer and filler composition Dental Materials 22(6) 515-526.

21. de Silva AL, Lima DA, de Souza GM, dos Santos CT \& Paulillo LA (2006) Influence of additional adhesive application on the microtensile bond strength of adhesive systems Operative Dentistry 31(5) 562-568.

22. Faria-e-Silva AL, Casselli DS, Lima GS, Ogliari FA, Piva E \& Martins LR (2008) Kinetics of conversion of two dual-cured adhesive systems Journal of Endodontics 34(9) 1115-1118.

23. Shafiei F, Safarpoor I, Moradmand M \& Alavi AA (2009) Effect of light activation mode on the incompatibility between one-bottle adhesives and light-cured composites: An in vitro shear bond strength study Operative Dentistry 34(5) 558-564.

24. Tay FR, Suh BI, Pashley DH, Prati C, Chuang SF \& Li F (2003) Factors contributing to the incompatibility between simplified-step adhesives and self-cured or dual-cured composites Part II. Single-bottle, total-etch adhesive Journal of Adhesive Dentistry 5(2) 91-105.

25. Pfeifer C, Shih D \& Braga RR (2003) Compatibility of dental adhesives and dual-cure cements American Journal of Dentistry 16(4) 235-238.
26. Caughman WF, Chan DC \& Rueggeberg FA (2001) Curing potential of dual-polymerizable resin cements in simulated clinical situations The Journal of Prosthetic Dentistry 86(1) 101-106.

27. Moraes RR, Faria-e-Silva AL, Ogliari FA, Correr-Sobrinho L, Demarco FF \& Piva E (2009) Impact of immediate and delayed light activation on self-polymerization of dual-cured dental resin luting agents Acta Biomaterialia 5(6) 20952100.

28. Ye Q, Spencer P, Wang Y \& Misra A (2007) Relationship of solvent to the photopolymerization process, properties, and structure in model dentin adhesives Journal of Biomedical Materials Research Part B: Applied Biomaterials 80(2) 342350.

29. Arrais CA, Giannini M \& Rueggeberg FA (2009) Kinetic analysis of monomer conversion in auto- and dual-polymerizing modes of commercial resin luting cements The Journal of Prosthetic Dentistry 101(2) 128-136.

30. Feng L \& Suh BI (2006) The effect of curing modes on polymerization contraction stress of a dual cured composite Journal of Biomedical Materials Research Part B: Applied Biomaterials 76(1) 196-202.

31. Fruits TJ, Knapp JA \& Khajotia SS (2006) Microleakage in the proximal walls of direct and indirect posterior resin slot restorations Operative Dentistry 31(6) 719-727.

32. Duquia Rde C, Osinaga PW, Demarco FF, de V Habekost L \& Conceição EM (2006) Cervical microleakage in MOD restorations: In vitro comparison of indirect and direct composite Operative Dentistry 31(6) 682-687.

33. de Andrade OS, de Goes MF \& Montes MA (2007) Marginal adaptation and microtensile bond strength of composite indirect restorations bonded to dentin treated with adhesive and low-viscosity composite Dental Materials 23(3) 279-287. 